\title{
Une succession de vagues involutées
}

\section{Alain Froidevaux}

\section{OpenEdition}

Journals

Édition électronique

URL : http://journals.openedition.org/ccs/1049

DOI : $10.4000 /$ ccs. 1049

ISSN : 2558-782X

\section{Éditeur :}

Presses universitaires de Rennes, Association des lecteurs de Claude Simon

\section{Édition imprimée}

Date de publication : 1 décembre 2017

Pagination : $129-144$

ISBN : $978-2-7535-5482-5$

ISSN : 1774-9425

\section{Référence électronique}

Alain Froidevaux, "Une succession de vagues involutées », Cahiers Claude Simon [En ligne], 12 | 2017, mis en ligne le 07 décembre 2018, consulté le 12 février 2020. URL : http://journals.openedition.org/ ccs/1049; DOI : 10.4000/ccs.1049 


\title{
UNE SUCCESSION DE VAGUES INVOLUTÉES
}

\author{
Alain FROIDEVAUX \\ Collège de Genève
}

À quelqu'un qui dirait: «je ne sais pas s'il y a là une main ", on pourrait dire: "Regardes-y de plus près »- cette possibilité de bâtir sa conviction appartient au jeu de langage, en est un des traits essentiels. Wittgenstein, De la certitude

Travaillant sur Les Géorgiques avec les archives de l'un de ses ancêtres sous la main, Claude Simon ne disait-il pas avoir éprouvé la stimulante impression de "toucher l'Histoire du doigt ${ }^{1}$ "? Dans le texte du roman, une expression comme celle-ci prend tout son sens dans la description d'une lettre imprimée sur

un de ces papiers épais au toucher, crémeux et légèrement cloqué, où la presse à main avait imprimé en creux, comme dans une matière impérissable (on pouvait les sentir en passant le doigt) les caractères en taille-douce, dessinés à la main, pas absolument réguliers et avec parfois dans les pleins de légères bavures, carrés, catégoriques ${ }^{2} .(G, \mathrm{p} .761)$

Point de contact entre mots et matériaux, entre deux modes d'être des choses, le doigt identifie la matière crémeuse, les traces typographiques légèrement cloquées de l'Histoire. Ce faisant, le toucher prolonge le regard d'un ail-

1. C. Simon, lettre à John Fletcher du 6 juin 1973, citée par A. B. Duncan dans la notice des Géorgiques, E II, p. 1496. Simon utilise la même expression dans un entretien radiophonique lorsqu'il relate l'une de ses découvertes poétiques fondamentales: "Les Corps conducteurs sont sortis de la dernière partie de La Bataille de Pharsale où j'avais vraiment touché du doigt, d'une façon tout à fait consciente, pour la première fois, qu'on n'écrit jamais que ce qui se passe au présent de l'écriture " (Écrivains du $X X^{e}$ siècle, France Culture, 1/3, 4 déc. 2014).

2. Sauf mention contraire, ici et dans la suite, les italiques sont de Simon. 


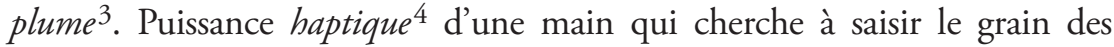
choses, comme le dit bien Goethe, dans sa sixième Élégie romaine, où le poète palpe le marbre d'une statue: "Dann versteh ich den Marmor erst recht; ich denk und vergleiche, / Sehe mit fülhendem Aug, fühle mit sehender Hand ${ }^{5}$. "

Lorsqu'il s'agit de redonner "forme au sensible ${ }^{6}$ ", les sens sont appelés à collaborer à l'ouvrage. Dans les Géorgiques, lorsqu'à l'occasion de travaux de réfection, l'on découvre sous des couches de papier peint le réduit où se trouvent serrés les registres du conventionnel et général d'Empire Jean-Pierre L.S.M., les " langues pendantes ${ }^{7}$ » de la tapisserie délabrée se détachant " dans une écœurante odeur de vapeur, d'étuve et de vieille colle chauffée» (p. 934) sollicitent aussi bien l'olfaction que le tact dans la prise en main des archives de cette figure familiale tutélaire. Au toucher des caractères en taille-douce d'un document imprimé correspond une "odeur de renfermé, de passé prêt à resurgir, sinon intact du moins palpable, irrécusable comme le monumental buste de marbre lui-même ${ }^{8}$ "(p. 938). Attentive à l'irrécusable présence de la matière dans laquelle est sculptée cette figure familiale tutélaire, à la texture des papiers peints ou imprimés, la poétique simonienne ne cesse de rendre justice à cette " chair du monde » qui nous enveloppe. Et si, de prime abord, la matière comprend "tout ce qui se touche et a corps et forme " (Dictionnaire Littré), l'écriture appliquée à manipuler notre fonds commun sédimenté dans le dictionnaire ne manquera pas de faire, comme on dit, flèche de tout bois. En effet, avec l'écriture, tout passe par la main. «Faire

3. Tel celui que l'on peut voir dessiné en marge d'une page manuscrite de La Bataille de Pharsale (voir L. Dällenbach, Claude Simon, Le Seuil, 1988, p. 68).

4. C'est à l'historien d'art Aloïs Riegl qu'Henri Maldiney (Regard, parole, espace, Lausanne, L'Âge d'homme, 1973) et Gilles Deleuze (Francis Bacon logique de la sensation, Le Seuil, 2002) empruntent cette catégorie esthétique.

5. «Alors je puis vraiment comprendre le marbre; je réfléchis/Et compare; alors l'œil palpe, la main y voit ", Goethe, Élégies romaines, VI. Un geste que Jean Starobinski commente en ces termes: «les mains veulent voir, les yeux souhaitent caresser. À quoi l'on peut ajouter: le regard veut devenir parole, il consent à perdre la faculté de percevoir immédiatement, pour acquérir le don de fixer plus durablement ce qui le fuit " (J. Starobinski, L'Eil vivant, Gallimard, "Tel », 1991 p. 12).

6. Comme le suggère le beau titre donné par Judith Sarfati-Lanter à son essai d'esthétique phénoménologique comparée: Donner forme au sensible. La perception dans l'œuvre de Peter Handke, Malcolm Lowry et Claude Simon, Champion, 2013.

7. Ces langues pendantes se trouvent déjà dans l'incipit du générique de Leçon de choses où la description d'une tapisserie décollée d'un local en ruine " où se répète le même motif (frise?) de feuilles d'acanthe dessinant une succession de vagues involvées ». L'édition de la Pléiade corrige ce néologisme (ou cette coquille) par l'adjectif d'usage: involuté ( $L C$, p. 557).

8. Jean-Yves Laurichesse s'attache au sens de l'olfaction dans La Bataille des odeurs, l'espace olfactif des romans de Claude Simon, L'Harmattan, 1998. 
est le propre de la main ", pouvait dire Valéry en ajoutant : " il faut aussi des mains pour instituer un langage ${ }^{9} "$.

Le motif d'une main au travail est visible dans des dessins de Claude Simon comme celui mis au frontispice d'Orion aveugle. Entre autres choses, devant une fenêtre, on remarque une main tenant un porte-plume. Mis en œuvre dans une "série 10 " de la première partie des Géorgiques où il intervient à douze reprises, ce motif n'aura pas manqué de retenir l'attention de la critique. Ainsi Jean Rousset s'intéresse à la main de l'écrivain, à «son outil de travail qui est en même temps son image miniaturisée ", un écrivain engagé dans une "empoignade " avec son ancêtre, l'un et l'autre pris dans une opération où ils "vont s'engendrer mutuellement dans un violent corps-à-corps ${ }^{11}$ ». Qu'ajouter à cette belle lecture sensible à la puissance régénératrice d'une écriture comme celle de Claude Simon ${ }^{12}$ ?

Ce corps à corps et cet engendrement, qui se jouent au présent d'une écriture, dans la lenteur de l'acte matériel d'écrire, impliquent une manière de faire, une gestuelle. Gestes de l'écriture comme ceux de sélection et de composition, privilégiés dans cette poétique. Gestes fictifs également: dès les premières pages des Géorgiques déjà, la description prête une attention soutenue à la facture du dessin mais aussi bien aux mains "pour ainsi dire gantées de peau humaine" (p. 652). Quelle que soit la perspective prise pour lire ce "prologue ", nous aurons d'une manière ou d'une autre à faire avec la prégnance de ce motif manuel ${ }^{13}$. En considérant cette série de la

9. Paul Valéry dans son "Discours aux chirurgiens" où il rendait hommage à l'art de la chirurgie: " manuopera, manœuvre, œuvre de la main " (Euvres I, Gallimard, "La Pléiade ", 1957, p. 918).

10. "Série " est le terme que l'écrivain lui-même utilise pour qualifier un élément textuel susceptible de fonctionner dans ses "plans de montage " (M. Balcázar Moreno, "Histoire et lisibilité dans les manuscrits des Géorgiques ", dans M. Calle-Gruber, M. Balcázar Moreno, S.-A. Crevier Goulet et A. Frantz (dir.), Les Vies de l'Archive, Dijon, EUD, «Écritures », 2014, p. 97-107).

11. J. Rousset, "Usure et rajeunissement dans Les Géorgiques ", Passages échanges et transpositions, José Corti, 1990, p. 177 - une lecture que Bernard Andrès peut mettre à profit dans son interprétation des derniers romans simoniens comme " "alterbiographie" d'un ego recouvré " chez un auteur assumant "la biographie de son alter ego romanesque " (Profils du personnage chez Claude Simon, Minuit, 1992, p. 214).

12. La génétique vient confirmer cette hypothèse avec ces extraits des manuscrits: "le "présent" le narrateur qui s'identifie peu à peu au Général " ou encore "le parallèle entre le Général et l'écrivain - qui s'inscrit ici explicitement dans l'univers de la fiction ("Moi à ma table de travail regardant lettres/ le Général sur sa terrasse le jour de sa mort") ", M. Balcázar Moreno, art. cité, p. 100 (n. 6) et p. 101.

13. Mise en abyme de la fiction et du code (B. Ferrato-Combe, Écrire en peintre, Claude Simon et la peinture, Grenoble, Ellug, 1998, p. 95-103), critique d'un art idéaliste du dessin (M. Créac'h, "Le prologue des Géorgiques: une théorie du dessin? ", Cahiers Claude Simon, n 9, 2014). 
main comme une sorte de leçon d'anatomie de l'écriture, nous chercherons à répondre de l'affectivité d'une pensée inscrite dans le moindre geste ${ }^{14}$.

\section{MATÉRIALISMES}

"Grosso modo, je suis matérialiste », concédait Simon dans une interview de 1989. Et c'est l'un des intendants du Jardin des Plantes qu'il donne alors en exemple:

Il y a un texte extraordinaire de Buffon [qui] décrit Adam au moment où il s'éveille au monde: il voit le ciel, les nuages, les arbres, il entend le chant des oiseaux et il croit que tout cela c'est lui-même jusqu'au moment où en étendant la main il touche quelque chose et prend seulement alors conscience qu'un univers solide l'entoure, extérieur à lui ${ }^{15} \ldots$

Ce résumé d'une page de Buffon consonne avec les expériences de primordialité vécues par des personnages comme le brigadier de L'Acacia (après l'épisode de la tranchée) ou par Gastone Novelli au cœur de la forêt amazonienne dans Le Jardin des Plantes. Dans Les Géorgiques, c'est à Jean-Marie, le frère hors-la-loi de L.S.M. et au cavalier défait de 40, qu'il revient de vivre une telle expérience. Lors d'un épisode de la débâcle, ce dernier n'éprouve-t-il pas précisément l'impression d'être retourné à l'état de "ces organismes à mi-chemin entre le poisson, le reptile et le mammifere qui à l'aube du monde, avant la séparation des terres et des eaux, se traînaient dans la vase en s'aidant de choses elles aussi à mi-chemin entre deux noms: déjà plus des nageoires et pas encore des membres" ( $G$, p. 924, nous soulignons)? Dans ce retour à une étape d'avant le langage où la main ne s'est pas encore développée, on conçoit bien que "plus rien n'y avait la même signification, sinon de signification tout court" (p. 926). Sauf peut-être la vie qui, dans sa puissance, continue de se manifester par le coït en vol de deux libellules, "les deux paires d'ailes de mica continuant toujours à frémir, étincelantes, avec la même foudroyante rapidité" (ibid.).

Cette fable adamique illustre les débats passionnés des savants et des philosophes des XVII ${ }^{\mathrm{e}}$ et XVIII ${ }^{\mathrm{e}}$ siècles qui héritent du dualisme cartésien avec ses animaux-machines, ses automates réglés comme des horloges et ce Dieu qui, tel Pygmalion, anime de son feu la matière inerte du corps humain, selon le modèle théorique proposé par Descartes dans son Traité de l'Homme: "Je suppose que le corps n'est autre chose qu'une statue ou une machine de terre,

14. Voir M. Guérin, Philosophie du geste, Actes Sud, 2011.

15. «L'atelier de l’artiste », entretien avec Jean-Claude Lebrun, Révolution, 29 septembre 1989. 
que Dieu forme tout exprès, pour la rendre la plus semblable à nous qu'il est possible ${ }^{16}$. " Le mythe de Pygmalion (comme mythe d'origine de la sculpture $^{17}$ ) ou l'allégorie de la statue (comme expérience de pensée) regardent les questions de métaphysique et de psychologie qui occupaient les bons esprits d'un siècle sensualiste: Bonnet, Deslandes, Condillac, Rousseau, Diderot... Chez Buffon, dans la conclusion du deuxième tome de son Histoire naturelle, cette expérience de pensée prend le tour de la prosopopée au moment où le "traité s'incarne en récit " remarque Aurélia Gaillard, lorsqu’il s'agit de montrer la spécificité de l'homme au sein d'un système matérialiste de la nature $^{18}$. Et, ajoute-t-elle, « le corps statufié quitte son statut de pur objet épistémologique pour devenir un personnage de fiction qui s'anime ${ }^{19}$ " dans toute une série d'expériences perceptives. C'est précisément à la main qu’il revient d'enrichir les premières perceptions visuelles d'une figure spéculative sculptée par un styliste. Cet organe donne tout d'abord à l'esprit une idée de corps puis, dans un deuxième temps, l'idée de son propre corps et de la conscience de soi. Marchant "la tête haute levée vers le ciel ", voici que le premier homme se heurte à un arbre. C'est un palmier ${ }^{20}$ qui met fin au solipsisme et instaure une limite entre les choses matérielles et la conscience d'Adam: " et je connus, pour la première fois, qu'il y avait quelque chose hors de moi $^{21}$ ".

On a pu reconnaître Lucrèce comme l'une des grandes références philosophiques des Géorgiques ${ }^{22}$. Mais il y en a bien d'autres si l'on tient

16. Le Traité de l'Homme commence ainsi sous l'égide d'une statue (Descartes, Euvres philosophiques, Garnier, 1963, t. I, p. 379).

17. J. Starobinski, "Le regard des statues ", L'Encre de la mélancolie, Le Seuil, 2012. Voir également, J. Larfouilloux, Perceptions philosophiques occidentales sur la sculpture et ses techniques de Socrate à Hegel, Éditions Arguments, 2000.

18. Du matérialisme de Buffon, François Dagognet peut dire ceci: «Jamais philosophe n’est allé aussi loin sur la voie d'une métaphorisation unitaire illimitée: pour ce savant, il ne faut d'ailleurs en fin de compte qu'un ressort, une seule force, qui engendre tout le reste, la multiplicité phénoménale, son mouvement, l'agitation des particules ", Pour une théorie générale des formes, Vrin, 1975, p. 41.

19. A. Gaillard, Le Corps des statues, Le vivant et son simulacre à l'âge classique (de Descartes à Diderot), Champion, 2003, p. 91.

20. C'est un palmier «aux feuilles en forme d'éventails et pointues comme des épées, au tronc d'un brun foncé, d'une matière rêche, comme du crin " (JP, p. 1073), que l'on trouve dans la cour de la maison familiale où le narrateur du Jardin des Plantes et sa jeune cousine vont jouer après le déjeuner réunissant la famille à la mort de la mère.

21. Buffon, Histoire naturelle, générale et particulière de l'Homme, "Des sens en général ", Euvres, Gallimard, «La Pléiade », p. 304.

22. Entre autres, Pierre Macherey pour qui la «grande référence philosophique qui, implicitement, se tient à l'arrière-plan des Géorgiques de Simon [...], c'est Lucrèce, qui déjà avait inspiré Virgile, et 
compte des contemporains d'un personnage, L.S.M., tout à la fois mathématicien, pamphlétaire et maquignon, impliqué dans les disputes d'un siècle où, dans un contexte intellectuel polémique, s'invente le terme de matérialisme ${ }^{23}$. Ces savants philosophes, eux aussi, entrent dans la matière des archives de l'Ancêtre, (comme sur les rayons de la bibliothèque familiale $^{24}$ ): Jean-Jacques Rousseau est présent dès le seuil du roman ${ }^{25}$, avec une épigraphe extraite du Livre Ix des Confessions. Jean-Jacques et L.S.M. ont non seulement tous deux séjourné à Montmorency mais l'esthétique, l'éthique et la politique de l'auteur du Contrat social conviennent aux idéaux civilisateurs du conventionnel, comme le précise l'oncle Charles en rappelant à son neveu que les chevaux, "le Contrat social et Virgile " $G$, p. 939) semblent avoir été les seules passions du Général.

Comme la statue de Condillac, le buste de L.S.M., un personnage ayant "labouré et moissonné les champs en esprit» $(G, \mathrm{p} .885)$, semble lui aussi donner matière à spéculation à ses héritiers. Le romancier peut non seulement en faire une idée directrice de la composition du roman ${ }^{26}$, mais cette entité colossale donne à l'écriture l'occasion de remettre en jeu l'une des questions irritantes du matérialisme, celle de la relation entre la matière et l'esprit ${ }^{27}$.

c'est aussi avec lui toute la tradition des matérialistes de l'Antiquité, qui avaient développé la vision d'une nature en proie à des soubresauts et à des dérives associant en permanence conservation et altération " [http//philolarge.hypotheses.org/482].

23. "Même si les thèses matérialistes remontent à l'antiquité, nous rappelle Franck Salaün, on peut considérer que le matérialisme a été inventé au $\mathrm{XVIII}^{\mathrm{e}}$ siècle et mis en évidence pour des buts spécifiques. " Pour mesurer les enjeux philosophiques de cette invention, voir "Anti-matérialisme et matérialisme en France vers 1760 ", dans J.-C. Bourdin (dir.), Les Matérialismes philosophiques, Kimé, 1997, p. 113-132.

24. Ce sont "les œuvres complètes de Jean-Jacques Rousseau et l'Histoire des Girondins en plusieurs volumes illustrés d'images" ( $J P$, p. 1072) qui garnissent les rayons de la bibliothèque de la chapelle ardente où se trouve le cercueil de la mère.

25. Sur le rôle de l'œuvre de Rousseau dans la critique simonienne de l'humanisme, voir F. DugastPortes, «Le spectre de l'ascendance: fonction tragifiante du personnage de l'ancêtre au fil de l'œuvre de Claude Simon ", Revue des Sciences Humaines, n² 215, 1989, p. 201-220.

26. Dans le processus de l'écriture, ce buste est d'abord un élément de composition: Simon n'intitule-t-il pas justement "buste " l'une des parties du roman en cours (voir le feuillet manuscrit des Géorgiques intitulé "Collages dans "Le buste" ", cité dans M. Balcazár Moreno, art. cité, p. 103).

27. Sur la question disputée du matérialisme, voir Vincent Descombes, "Remarque sur le matérialisme ", La Denrée mentale, Gallimard, 1995, p. 99-104. Voir également J. Proust, "Matérialisme et intentionnalité ", dans J.-C. Bourdin (dir.), op. cit, p. 173-196. 


\section{LES RIMES DE LA MAIN}

Cette matière première, cette glèbe dont se constituent les corps relève d'une thématique toute simonienne. Dans un entretien de 1958, le romancier n'employait-il pas déjà cette belle expression d' "humus de la matière " pour envisager l'analogie de la recherche scientifique et du travail romanesque? Simon trouvait bon de rappeler que la description de "personnages dans certaines situations - étant entendu que celles-ci ne sont pas seulement psychologiques ou sociales mais aussi physiques: assis, debout, dans une pièce, grande, petite, dehors, au soleil, à l'ombre, assoiffé, repu, etc. ", n'allait pas sans une opération de l'esprit, tout en soulignant que hors de ce vaet-vient permanent entre chose étendue et chose pensante, "l'écrivain court le danger de voir son esprit fonctionner dans le vide ${ }^{28}$ ». Voyons ce que la description d'une main peut donner à penser dans Les Géorgiques.

Dans la dernière année de sa vie, la main fripée de l'Ancêtre (p. 887) lui permet encore de récapituler son existence, de relire ses registres et d'y apporter d'ultimes corrections. Ridée, la main du " narrateur » ou du " scripteur " accomplit les mêmes gestes en manipulant les registres du colosse:

les doubles de centaines de lettres, de rapports, de mémoires, de bordereaux, de comptes de domestiques, de projets d'armées, d'inventaires d'arsenaux, d'ordres et d'instructions de toutes sortes, parfois des récits de voyages ou des brouillons de discours. ( $G$, p. 687)

La description de cette main donne textuellement corps à un motif de composition.

Cette série consacrée à la main d'un scripteur est spatialement repérable: une pièce meublée d'une table de travail devant une fenêtre. Comme, sur un plan temporel, avec le déplacement du soleil éclairant «d'un jour frisant la main qui feuillette les cahiers format registre aux pages couvertes d'une écriture régulière" (p. 657). Unité d'action avec la reprise (au moins au sens équestre du terme) d'un groupe de cavaliers et de leurs chevaux dans la cour d'une caserne qui suit la même chronologie: "Dans le contre-jour du soleil déclinant le jet de la lance scintille comme de l'argent. Les ombres de plus en plus en plus pâles du cheval et de l'homme s'étirent démesurément sur le pavé " (p. 687) cependant que « [l]a main ridée qui feuillette les registres se trouve maintenant entièrement dans l'ombre et la lumière diffuse luit faiblement sur la surface crêpelée de la peau, les saillies vert pâle et noueuses des veines »

28. C. Simon, "Un bloc indivisible », Les Lettres françaises, n 760, 4-10 décembre 1958, p. 5. 
(p. 687-688). Le scintillement de ce jet de la lance, lui, ne cesse de diffuser sa lumière dans le texte.

Au fil des pages, la main intervient "pour saisir le coin supérieur des pages entre le pouce et l'index" (p. 665), pour tourner "plusieurs feuilles d'un coup" (p. 664). Feuilletant les archives, elle avance de gauche à droite dans la lecture de documents. Surprise par une liste autographe des chevaux de L.S.M., la voici comme prise par la main de l'ancêtre, laquelle, "revenue trop vite en arrière a balayé l'encre encore fraîche ", en laissant sur la page une trace indicielle, une coulure semblable à « la crinière d'un cheval au galop flottant dans le vent» (p. 689). Ainsi en manipulant les archives, la main ne tourne pas simplement des pages, elle réactive des indices, elle répond esthétiquement à la présence d'une autre main.

"Cette main est philosophe », dirons-nous une fois encore avec Valéry ${ }^{29}$, lorsqu'il lui arrive de marquer un temps de réflexion; ainsi quand, écrivant, elle prend soudain conscience du non-sens d'une phrase venue spontanément «Ils comprennent alors qu'ils sont tombés dans une embuscade et qu'ils vont presque tous mourir" (p. 672). À ce moment, la main s'arrête, se relève et ce geste correspond, dans l'écriture du roman, à celui de O. occupé à écrire le témoignage de sa propre expérience d'engagé volontaire sur le front de la guerre civile espagnole. C'est alors que O. s'arrête de raconter, doute du sens de son entreprise testimoniale: son effort ne semble valoir guère mieux que la prose convenue de ces pages de journaux éparpillés sur la moquette. O. les connaît par cœur mais il ne continue pas moins à les manipuler comme s'il avait besoin de

se convaincre qu'il avait bien lu, toucher de ses mains le papier, contempler les colonnes imprimées), puis le laissant retomber parmi les autres qui jonchaient le sol, en piles instables ou tels qu'ils lui avaient glissé des mains, c'est-à-dire à demi déployés, froissés, s'affalant comme des espèces de volatiles aux ailes grisâtres et cassées, inertes sous son regard empreint de cette méditative incrédulité (quoiqu'elle fût alors d'une nature différente) qu'il avait ressentie, accroupi dans la boue ( $G$, p. 870 ; nous soulignons)

Ces gestes ne sont à proprement parler ni ceux d'un personnage en action, ni ceux d'une main qui écrit. Ils ne correspondent ni à un acte politique (praxis) ni à une activité productrice (pö̈étique). De tels gestes de retrait ouvrent un temps de réflexion commun à chacun de ces trois personnages. Dans une note consacrée à une ontologie du geste, Giorgio Agamben envisage trois modalités de l'action: il distingue l'action pratique, le faire poïétique et le caractère pro- 
prement éthique de la gestualité. Un geste selon lui, c'est étymologiquement une manière de porter et de prendre sur soi l'événement dont on devient le vecteur. "Autrement dit, le geste ouvre la sphère de l'éthos comme sphère la plus propre de l'homme ${ }^{30}$. " Lorsque survient une expression esthétiquement ou éthiquement inqualifiable, la main engagée dans la manipulation d'un document, la main employée à écrire laisse tomber, se tient suspendue, désigne l'incrédulité empreinte dans le regard de $\mathrm{O}$. ou "l'empreinte lumineuse laissée sur la rétine par le rectangle du cahier ouvert" (p. 665) du registre sur la table de travail. Un tel geste donne à penser et suscite une forme de méditation où surviennent $\mathrm{d}$ ' " inapaisables fantômes " dans le dos de l'écrivain anglais; où se configurent de "vagues formes noires" qui (mais faut-il le souligner) se succèdent à travers les volutes de fonte du balcon d'une pièce de travail ouverte sur la cour d'une caserne.

En s'élevant son regard rencontre successivement le haut de la feuille de papier couverte de ratures, le bord de la table, puis les volutes et les feuillages de fonte du balcon au-delà desquels continuent à se succéder les vagues formes noires. $(G$, p. 672$)$

Comme le diaphragme d'un appareil photographique ${ }^{31}$ (ou cinématographique), les yeux clignotent, s'ouvrent et se ferment machinalement, cependant que " $[l a]$ tache du registre aux pages éclairées par le soleil reste imprimée sur sa rétine" (p. 658). C'est sur un plan modal que nous examinerons la configuration esthétique de ces empreintes rétiniennes ${ }^{32}$.

\section{VAGUES}

Des séquences en focalisation zéro (comme celle où la lumière frisante du soleil met en lumière le geste de manipulation des archives) alternent avec des séquences en focalisation interne où sont exposées les impressions de la main ridée et des yeux fatigués par le travail. Ainsi par exemple:

Dans le mouvement quil fait pour saisir le coin supérieur des pages entre le pouce et l'index les rides et les saillies des veines séffacent et la peau se tend sur le dos de la main qui semble

30. G. Agamben, "Notes sur le geste", Moyens sans fins, Notes sur la politique, Payot-Rivages, 2002, p. 68. Selon André Leroi-Gourhan, en se libérant des contraintes pédestres, la main «apanage de l'homo faber, instrument du cerveau le mieux organisé de la série zoologique, libre des contraintes pédestres est le symbole de l'évolution de l'homme ", la main indique la question de la destination de l'homme ("Libération de la main ", Problèmes, n³ 32, 1956, p. 6).

31. Cette comparaison peut nous être suggérée par le narrateur du Vent interloqué par la conduite émotionnelle singulière de Montès et la puissance photographique de son regard ( $V, \mathrm{I}, \mathrm{p} .36)$.

32. J.-M. Schaeffer, L'Expérience esthétique, Gallimard, 2015. 
alors fait d'un marbre lisse et rosé parcouru d'un pâle lacis bleuâtre. ( $G$, p. 665 ; nous soulignons par des romains)

L'adverbe alors instaure le point de vue d'un corps attentif à la présence de sa main. Mais la composition marmoréenne de cet organe nous indique que ce pourrait tout aussi bien être celle du colosse de marbre.

À l'interruption de la manipulation des cahiers, succède le regard scrupuleux que le personnage accorde à cette main exposée au soleil «qui fait ressortir les milliers de rides plus ou moins larges se chevauchant, s'entrecroisant, mais toutes orientées dans le même sens " (p. 660). Le chevauchement de ces rides correspond aux innombrables chevauchées de l'Ancêtre, sans oublier celles de bien d'autres cavaliers. Ce chevauchement ne dit-il pas tout ce que cette main aura pu incorporer de la matière des registres aux «dos renforcés de parchemin» (p. 687) ? Il y a toute une histoire naturelle dans ces rides qui font " comme des plissements de terrain " (ibid.). Du tranchant de la main - rose comme « les tranches des épais registres [...] d'un rose fané » (ibid.) -, celles-ci progressent obliquement vers l'index. Il y a sans doute dans ces rides, qui évoluent en sillonnant la peau du texte, une déferlante de vagues "ondulant, se resserrant ou s'écartant, s'engouffrant entre les bases des doigts comme l'eau d'un courant» (p. 660), un flux de possibilités descriptives. Tout à fait littéralement, le vague et la vague entrent dans la texture des formes noires que le personnage voit d'abord confusément. Mais voyons plutôt:

Au-delà du cabier ouvert sur la table et des volutes du balcon il peut voir en contrebas la cour de la caserne où se succèdent les cavaliers. Ceux-ci sont vêtus de tuniques noires. Sur la page du registre le nom de Moustapha ainsi que les trois lignes de son signalement sont barrés de traits obliques. (G, p. 660)

Cette vision se prolonge dans le texte quelques pages plus loin:

À travers les volutes et les feuilles de fonte du balcon il continue à percevoir confusément au-dessous de lui les silhonettes obscures des cavaliers qui se succèdent sur le fond de lumière. [...] Les bustes vêtus de tuniques noires se dandinent légèrement au pas des chevaux. Leurs ombres de statues équestres sétirent sur le pavé selon le même angle que celle projetée sur le cahier par la main. ( $G$, p. 664)

À la troisième mention des volutes et des feuillages du balcon, au-delà du cadre de la fenêtre, le personnage retirant ses lunettes parvient à " voir avec netteté dans la cour de la caserne les cavaliers aux bustes droits vêtus de tuniques noires soutachées de rouge, avec leurs bottes noires et luisantes » (p. 672). 
Jeux d'ombres et de lumière, couleurs chatoyantes des éléments : admirable composition picturale de cette pièce de travail ouverte sur le dehors par une porte-fenêtre et un balcon. Dans cette succession des statues équestres, nous percevons les étapes d'une écriture descriptive manipulant avec précision des matériaux propres à s'harmoniser dans tout le roman. Ces formes noires procèdent certes de la lecture des archives mais n'affectaient-elles pas aussi bien L.S.M. dans les derniers jours de sa vie: "Après son attaque d'apoplexie il est parfois sujet à des étourdissements. Il voit tournoyer des points noirs " (p. 662)? Vol noir et discordant de corneilles perçu par un vieillard sur sa terrasse: "Un vol noir de corneilles tournoie au-dessus de la terrasse dans un lent battement d'ailes et un tapage de cris discordants. Il est fatigué. Il ferme les yeux» (p. 658). Ce vol entre dans la matière verbale des volutes du balcon. Tout comme le vol automnal des étourneaux qui succède à celui des corneilles, les choses laissent sur la rétine " des nuages pointillés " en permanente évolution:

Le nuage est plus foncé lorsqu'il se condense, presque noir, s'éclaircissant lorsqu'il s'allonge, file dans une direction ou l'autre, parfois au contraire comme suspendu sur place, immobile, nombreux et pour ainsi dire clignotant. ( $G$, p. 686)

Lorsque les yeux se ferment, le mouvement s'inverse et l'écriture s'attache alors à décrire ce que peut éprouver un regard aveuglé. "Sous ses paupières refermées les couleurs sinversent. Le rectangle vert jade de la fenêtre se scinde en deux rectangles cerise sur fond olive" (p. 666). Cette étonnante expérience kaléidoscopique demanderait un examen détaillé33. On s'en tiendra à la dernière apparition du phénomène: elle correspond, dans cette série, au déclin du soleil. "Il voit un soleil mauve sur fond brun. Le mauve se décolore, devient blafard, comme une lune indécise entourée peu à peu d'un halo plus clair qui se casse et se divise en marbrures, en polygones irréguliers" (p. 682). Une commune matière entre donc dans la composition des marbrures présentes sur la membrane rétinienne et sur la peau de la main relevée faite "de marbre lisse et rosé » (p. 665).

\section{LA MAIN PASSE}

Aussi vagues qu'elles puissent apparaître dans la description, ces perceptions visuelles conservent pourtant quelque chose d'aussi palpable et irrécu-

33. Le motif de l'empreinte rétinienne apparaît aux pages 658, 665-666-667, 673, 678, 682. 
sable que "le monumental buste de marbre lui-même ". À peine vient-elle d'arriver dans le texte que déjà cette figure colossale semble être prise dans la matière même de son buste, "un marbre gris ocre légèrement veiné de gris foncé » (p. 665), que l'Ancêtre fait donc exécuter par un sculpteur à Montmorency avec le désir de témoigner "pour la postérité du défi qu’il avait lui-même incarné, la tranquille détermination qu'il avait puisée dans la lecture de ces auteurs latins» (p. 772).

Avec un personnage comme L.S.M., les actes d'institution sont évidemment l'une des grandes affaires de ce colosse à la main grisâtre, dessinée, estompée, gommée mais toujours repérable lorsqu'elle se manifeste par un geste expressif tout à la fois "négligent et impérieux ${ }^{34}$ " (p. 653). Dès les premières pages du roman, c'est comme geste de la main que cette puissance régulatrice se manifeste. Cette main qui donne congé aussi bien commande, incite, exhorte, vote la mort d'un roi, feuillette des archives, les classe et les commente en de fertiles digressions, correspond avec d'autres acteurs de l'histoire, écrit des poèmes élégiaques etc. Ces différents gestes performatifs d'un Ancêtre cultivateur et guerrier sont ceux d'un homme éprouvé à l'art du discours. Cette puissance de la nature, sans cesse en train de faire signe, incarne la force constitutive de la parole qui (de Sieyès à Searle, pour faire court) institue la réalité sociale. Tout comme la lettre du prologue "que continue de tenir l'autre main " (ibid.) passe de main en main dans le roman, la "main détachée " se trouve elle aussi destinée à circuler en permanence dans le texte. Au terme de sa vie, victime d'une attaque d'apoplexie, le général finit certes par lâcher prise: « les bras fatigués laissant à la fin retomber le lourd registre " (p. 890). Sur le point de passer la main, ce corps usé reçoit enfin le silence, les voix infimes de la nature « ou plutôt ces menues manifestations du silence que sont les froissements des herbes, des feuilles, les invisibles cheminements d'insectes, cette confuse rumeur faite d'infimes bruissements, d'infimes palpitations " (p. 893; nous soulignons). Palpables, ces manifestations ne manquent pas de laisser leurs traces sur la vieille main fatiguée.

Mais comment concevoir qu'un buste puisse se trouver doté de mains? Dans le cours des successions, des ventes et des faillites familiales, le buste

34. Dans un essai qui prolonge l'anthropologie du geste dont se sont occupés Mauss, Jousse et LeroiGourhan dans Le Geste et la parole, Yves Citton dit de ce geste repérable qu'il est « une apparence qui se détache de nos actions pour gagner une agentivité [agency] propre dont se nourriront à la fois nos gestes et nos actions ultérieures " (Gestes d'humanités, Anthropologie sauvage de nos expériences esthétiques, Armand Colin, 2012, p. 56). 
finit tout de même par disparaître, même si cette chose ou cette cause paradoxale ne manque pas de laisser son empreinte sur la moquette de son dernier propriétaire, une empreinte semblable à " ces traces de pieds géants qu'on prétend laissées dans un rocher par quelque personnage fabuleux ${ }^{35}$ " (p. 802). Nathalie Piégay propose une intéressante et riche interprétation de la « revenance " de l'Ancêtre. Sur un plan métapsychologique, elle identifie l'emprise d'un "spectre sans existence réelle et pourtant pondérable » (p. 687) aux processus de la mélancolie (Freud) et de la hantise qui ne cessent d'obséder ses descendants. En ce sens, gardienne des secrets de famille, la grand-mère ne parvient pas à faire le deuil de cette figure tutélaire et semble être un bon exemple mélancolique. Mais, encryptée dans la demeure familiale, cette entité fantasmatique intime aussi bien " au petit garçon, puis à l'homme qui a fait la guerre à son tour [...] de prendre en compte les "lacunes" de son histoire [...] le secret de famille qui hante sa conscience». D'une certaine manière, c'est par la main d'un secrétaire (chargé d'exposer un secret), que ce fantôme contribue à ce que « la hantise [puisse] se transformer en énergie scripturale ${ }^{36}$ ».

Comme la tête d'Orphée ${ }^{37}$, la main de l'Ancêtre passe d'un corps à l'autre tout au long du roman. Lors d'une représentation d'Orphée à l'opéra où, dans un but éducatif, la grand-mère "aux mains veinées de bleu " emmène son petit-fils, il arrive que le programme lui échappe des mains (et l'incite peut-être à s'échapper de la forteresse familiale pour assister aux cérémonies tribales du cinéma). Des années plus tard, le garçon devenu à son tour un vieil homme visite le domaine en ruines de son ancêtre en compagnie de l'actuel propriétaire, une sorte d'idiot qui évoque le colosse en simulant « de ses mains [sa] carrure » (p. 747).

Cette puissance mimétique colossale est aussi bien puissance musicale si l'on prête attention aux improvisations de l'oncle Charles,

le buste se penchant parfois, se relevant, les longs doigts fins courant comme d'euxmêmes sur les touches d'ivoire, jouant de mémoire, sans partition sur le pupitre, ou improvisant, soutenant d'accords plaqués de la main gauche une lente mélodie dont l'autre détachait les notes $(G$, p. 794$)$

35. Voir A. Clément-Perrier, "L'empreinte et ses pouvoirs ", Littérature, n 147, 2007, p. 21-37.

36. N. Piégay, "La mélancolie des statues ", dans J.-Y. Laurichesse (dir.), Figure historique et personnage romanesque, Le général L.S.M. dans Les Géorgiques de Claude Simon, Littératures, n 73 , PUM, 2015, p. 114 et p. 115.

37. P. Schoentjes, Claude Simon par correspondance, "Les Géorgiques et le regard des livres, " Genève, Droz, 1995, p. 19. 
Cette pièce développée dans le troisième chapitre des Géorgiques est exécutée sous le regard "d'un impassible homme de pierre à l'impassible regard sans prunelles, condamné au silence et à l'obscurité » (p. 776).

Musicalement, la trace de ce regard silencieux se prolonge dans le jeu du pianiste. Elle sensibilise l'enfant qui revient de l'école à prendre rythmiquement possession d'une matière temporelle mémorable " que les notes pressées, se bousculant, se chevauchant et se disjoignant tour à tour semblaient tantôt précipiter, tantôt ralentir, disséquer...» (p. 795) Dans cette scène où le colosse de marbre se tient entre l'oncle et son neveu comme si, entre le pianiste et son jeune auditeur, "se tenait un troisième personnage ${ }^{38}$ " (p. 794), les trois points soulignent les résonances de l'accord plaqué de la main gauche par un oncle un peu artiste, un peu chimiste: les deux mains composent une polyphonie de voix qui se répondent à travers les épaisseurs du temps. Dans cette belle leçon de musique exécutée par un oncle qui manque rarement d'éclairer de ses commentaires historiques ou philologiques les difficultés que son neveu peut rencontrer dans son apprentissage, demeure pourtant une question intrigante si l'on prend garde au crissement de la plume audible dans le jeu silencieux du piano.

la plume du secrétaire grinçant dans le silence glacé, le colosse regardant paisiblement devant lui, attendant, la plume cessant de crisser (le piano jouant pour ainsi dire silencieusement aussi, lointain, feutré - en fait, maintenant, le garçon ne l'entendait plus), la voix de marbre s'élevant de nouveau, dictant ( $G$, p. 808)

À quelle instance en fin de compte convient-il d'identifier ce troisième personnage? Inscrite dans la mémoire d'un jeune garçon, cette dictée d'une "voix de marbre " réactive la plume d'un secrétaire congédié dans les dernières lignes $\mathrm{du}$ " prologue ", le geste d'une main qui ne cesse de recueillir, comme de l'écume laissée par les vagues sur le rivage, la précaire expérience des hommes.

\section{SUCCESSION}

Au terme de l'existence du général, « la vieille main à la peau fripée, piquetée de taches de sons» (p. 887; nous soulignons) s'acharne à couvrir de

38. L. Dällenbach: "l'archive fait mémoire de l'autre » en précisant qu' " elle concerne bien le sujet, mais dans un autre (que) lui-même: un proche dont ce sujet est irrémédiablement coupé et dont le passé (un certain passé en tout cas) lui demeurant insu, l'archive s'avérant la seule voie d'accès, toute conjecturale et problématique [...] à cette marge d'inconnu qu'il importe précisément de réduire ", ("L'Archive simonienne ", Claude Simon Colloquium, Modern Language Notes, vol. 103, n 4, p. 739). 
son écriture "d'abord impétueuse, violente, mal contrôlée, se désunissant peu à peu, vacillant» (p. 891), avant de cesser " de tracer des mots sur une feuille de papier ", pour sombrer "dans cet état en quelque sorte végétatif au sein duquel ne lui parvenaient plus que ces bruits minuscules de la campagne " (p. 892). Pourtant, tout comme les vagues ne cessent de se succéder, la dictée ne s'interrompt pas avec la rupture d'un vaisseau. L'évidence de "ces menues manifestations du silence ", l'attention aux infimes variations atmosphériques d'un vieux corps usé, la "tiédeur du soleil d'hiver, le parfum d'ozone de l'air glacé, les craquements ténus du gel» (p. 893), semblent à peine parvenir jusqu'au " cerveau mal irrigué » de l'Ancêtre mais ces sollicitations de la nature n'en continuent pas moins de mobiliser la main diligente d'un secrétaire ${ }^{39}$.

À point nommé, une mouche vient provoquer le mouvement réflexe de la main momentanément suspendue: "Il sent un léger frôlement sur le dos de sa main. Il rouvre les yeux» (p. 668). Mais à quel corps attribuer cette main aux "rides ondulant par-dessus les tendons et les veines saillantes" (ibid.) lorsqu' elle se remet à feuilleter les registres? Les doigts qui se déplient pour chasser l'insecte importun peuvent aussi bien être ceux de L.S.M. que les doigts du légataire de ces cahiers cousus mains.

Lorsque l'on tourne les pages des registres en les tenant inclinés de fines particules couleur rouille aux facettes scintillantes et dorées comme du mica se détachent des lettres et glissent sur les feuilles. On dirait que les mots assemblés, les phrases, les traces laissées sur le papier par les mouvements de troupes, les combats, les intrigues, les discours, s'écaillent, s'effritent et tombent en poussière, ne laissant plus sur les mains que cette poudre impalpable, couleur de sang séché. ( $G$, p. 692)

Ces pages conservent comme en dépôt quelque chose de la chair du monde et, dans le jeu ironique du texte l'on se prend alors à penser que tout le monde peut en devenir le dépositaire. La succession de phrases courtes et $\mathrm{du}$ pronom de la troisième personne en ouverture du roman ne donnet-elle pas au lecteur l'impression qu'il se trouve lui-même installé devant une fenêtre en train de feuilleter le roman, tout en cherchant à s'y repérer? Comme, par exemple, dans cette séquence où nous sont donnés à lire de manière inversée les fragments de vers de L.S.M., recouverts par " [l]ombre bosselée de la main qui s'étire»(p. 663), nous pouvons aussi bien tourner

39. Cette forme de mobilisation nous occupe dans une étude de L'Acacia, "Une mobilisation continuée », Cahiers Claude Simon, n 11, 2016, p. 111-133. 
"plusieurs feuilles d'un coup»(p. 664) que nous employer à recomposer l'élégie d'un humaniste inconsolable.

S'appliquer à entendre le silence dans les bruits du monde, à noter la présence d'insectes dont les ailes transparentes "brillent au soleil comme $d u$ mica» (p. 668), à observer les "toiles scintillantes et polygonales " qu'en septembre les grosses araignées tissent «d'une branche à l'autre " (p. 677), à mesurer en géomètre l'ombre portée d'une main sur la page, n'est-ce pas là une nouvelle occasion de pouvoir tirer parti de l'humus d'une culture commune? Dans le Rêve de d'Alembert, Diderot soutient l'hypothèse de l'unité de la matière et de l'esprit, de l'homme et de la statue, du marbre et de la chair. Cette idée, il se propose de la vérifier en réduisant en poussière une statue de Falconet: " lorsque le tout s'est transformé [...] en humus, j’y sème des pois, des fèves, des choux, d'autres plantes légumineuses. Les plantes se nourrissent de la terre et je me nourris des plantes ${ }^{40}$ ". Cet exercice de pensée spinoziste pourrait bien être la meilleure part du legs de l'Ancêtre. Il nous prédispose à recevoir comme il convient tout ce qui est, tout ce qui se présente: en prenant le temps de le lire.

40. Diderot, Le Rêve de d'Alembert, Euvres philosophiques, Garnier, 1964, p. 263. Olivier Asselin commente cette transformation dans "Le marbre et la chair: le modèle tactile dans l'esthétique matérialiste de Diderot ", Études françaises, vol. 42, n 2 2, 2006, p. 18-19. 